\title{
Correlação entre aumento do índice de massa corporal e diminuição da função pulmonar em crianças e adolescentes: uma revisão sistemática
}

\author{
Correlation between increased body mass index and reduced lung function in children and
}

adolescents: a systematic

Correlación entre el aumento del índice de masa corporal y la función pulmonar reducida en niños

y adolescentes: un estudio sistemático

Recebido: 12/01/2022 | Revisado: 16/01/2022 | Aceito: 16/02/2022 | Publicado: 25/02/2022

\author{
Morgana Thaís Carollo Fernandes \\ ORCID: https://orcid.org/0000-0002-7989-294X \\ Pontifícia Universidade Católica do Rio Grande do Sul, Brasil \\ E-mail: morganafernandes@yahoo.com.br \\ Marcos Otávio Brum Antunes \\ ORCID: https://orcid.org/0000-0001-8873-2385 \\ Pontifícia Universidade Católica do Rio Grande do Sul, Brasil \\ E-mail: marcos.antunes@edu.pucrs.br \\ Alexander Quadros \\ ORCID: https://orcid.org/0000-0002-3023-7514 \\ Faculdades Integradas de Taquara, Brasil \\ E-mail: alexanderquadros2005@yahoo.com.br \\ Frederico Friedrich \\ ORCID: https://orcid.org/0000-0001-6939-0340 \\ Pontifícia Universidade Católica do Rio Grande do Sul, Brasil \\ E-mail: fredericofriedrich@gmail.com
}

\begin{abstract}
Resumo
Objetivo: Avaliar os estudos acerca da relação entre o índice de massa corporal (IMC) e a função pulmonar em crianças e adolescentes. Métodos: Foram analisados estudos com ou sem diagnóstico de asma, sendo comparado ou associado à função pulmonar e ao índice de massa corporal (IMC) de delineamentos transversais, coortes, casoscontrole e ensaios clínicos randomizados (ECRs). Os dados foram selecionados utilizando-se os descritores (("Body Mass Index" OR BMI OR "Body Composition" OR "Body Fat Percentage" OR "Fat Percentage") AND Asthma AND ("Lung function" OR Spirometry)) nas bases de dados PUBMED, EMBASE, LILACS, SCIELO. Resultados: Foram incluídos 20 artigos na revisão sistemática. Do total, 10 artigos 5\% encontraram correlações entre o aumento do IMC e alterações na função pulmonar (VEF1). Dos que encontraram correlações, $5(50 \%)$ relataram correlação com a diminuição do VEF1 e o restante com o aumento do VEF1. Quanto a CVF, 53\% não possui correlação com o desfecho. Em relação ao VEF1/CVF 44\% não encontrou associação. Por fim, apenas $40 \%$ correlacionaram o >IMC com o FEF $25-75 \%$, sendo que $25 \%$ apresentaram diminuição do FEF $25-75 \%$. Conclusões: Apesar da maioria dos estudos ter indicado que o aumento do IMC tende a uma diminuição dos valores pulmonares, tais resultados e conclusões devem ser avaliados com cautela.
\end{abstract}

Palavras-chave: Sobrepeso; Índice de Massa Corporal; Crianças.

\begin{abstract}
Objective: To evaluate studies on the relationship between body mass index (BMI) and lung function in children and adolescents. Methods: Studies were selected with or without a diagnosis of asthma, being compared or associated with lung function and body mass index (BMI) of cross-sectional designs, cohort, case-control and randomized controlled trials (RCTs). The data were selected using descriptors (("Body Mass Index" OR BMI OR "Body Composition" OR "Body Fat Percentage" OR "Fat Percentage") AND Asthma AND ("Lung function" OR Spirometry)) on the basis of data PUBMED, EMBASE, LILACS, SCIELO. Results: We included 20 articles in the systematic review. Of the total, 10 items 5\% found correlations between increasing BMIand changes in lung function (FEV1). Of those found correlations, $5(50 \%)$ reported correlated with the decrease in FEV1 and the remainder with the increase in FEV1. The FVC, 53\% has no correlation with the outcome. Regarding the FEV1/FVC ratio $44 \%$ found no association. Finally, only $40 \%$ correlation between the >IMC with $\mathrm{FEF} 25-75 \%$, and $25 \%$ had decreased $\mathrm{FEF} 25-75 \%$ to >BMI. Conclusions: Although most studies have indicated that increased BMI tends to a decrease in lung values, these results and conclusions should be evaluated with caution.
\end{abstract}

Keywords: Overweight; Body Mass Index; Children. 


\begin{abstract}
Resumen
Objetivo: Evaluar estudios sobre la relación entre el índice de masa corporal (IMC) y la función pulmonar en niños y adolescentes. Métodos: Se analizaron estudios con o sin diagnóstico de asma, comparando o asociando la función pulmonar y el índice de masa corporal (IMC) en diseños transversales, cohortes, casos y controles y ensayos clínicos aleatorizados (ECA). Los datos fueron seleccionados utilizando los descriptores ("Índice de Masa Corporal" O IMC O "Composición Corporal" O "Porcentaje de Grasa Corporal" O "Porcentaje de Grasa") Y Asma Y ("Función Pulmonar" O Espirometría)) en las bases de datos PUBMED, EMBASE, LILAS, SCIELO. Resultados: 20 artículos fueron incluidos en la revisión sistemática. Del total, el 5\% de 10 artículos encontraron correlaciones entre el aumento del IMC y los cambios en la función pulmonar (FEV1). De los que encontraron correlaciones, 5 (50\%) reportaron correlación con disminución del FEV1 y el resto con aumento del FEV1. En cuanto a la FVC, el 53\% no tiene correlación con el resultado. Respecto al FEV1/FVC, el 44\% no encontró asociación. Finalmente, solo el $40 \%$ correlacionó >IMC con FEF25-75\%, y el 25\% tuvo una disminución de FEF25-75\%. Conclusiones: Aunque la mayoría de los estudios han indicado que un aumento del IMC tiende a una disminución de los valores pulmonares, tales resultados y conclusiones deben evaluarse con cautela.
\end{abstract}

Palabras clave: Sobrepeso; Indice de Masa Corporal; Niños.

\title{
1. Introdução
}

O sobrepeso caracteriza-se pelo acúmulo anormal ou excessivo de gordura corporal com etiologia complexa e multifatorial, resultado da interação de genes, do estilo de vida, do ambiente e fatores emocionais que pode levar a problemas de saúde aos indivíduos acometidos (Sigulem, 2001; ABESO, 2010). Reconhecido como um grave problema de saúde pública, a obesidade apresenta relevância a nível mundial alcançando proporções epidêmicas nos últimos anos em crianças e adolescentes (Alwan, 2011).

Dentre os comprometimentos à saúde oriundos do aumento de peso, os principais são cardiovasculares, endócrinos, gastrointestinais, ortopédicos e respiratórios (Koening, 2001; Schwimmer, 2003; Suchindran, 2010). Do ponto de vista biomecânico, o sobrepeso pode gerar alterações estruturais da região torácica e abdominal predispondo a limitação da mobilidade do diafragma e, consequentemente, do movimento costal degradando à mecânica ventilatória (Bekkers, 2015).

Estudos vêm tentando elucidar a influência do aumento do índice de massa corporal na Capacidade Vital Forçada $(\mathrm{CVF})$, Volume Expiratório Forçado no primeiro minuto $\left(\mathrm{VEF}_{1}\right)$, da "relação VEF1/CVF" e Fluxo expiratório forçado 25-75\% (FEF25-75\%), porém, não há consenso entre os achados, uma vez que alguns identificaram aumento dos elementos da função pulmonar e outros a diminuição dos mesmos (Pérez-Padilla, 2006; Ulger, 2006; Eisenmann, 2007; Chow, 2009; He, 2009; Spathopoulos, 2009; Paralikar, 2012; Bekkers, 2013; Han et al., 2014; Bekkers, 2015). Diante disso, se propôs a presente revisão sistemática analisar a Influência do Índice de Massa Corporal na função pulmonar de crianças e adolescentes.

\section{Metodologia}

\section{Delineamento}

Revisão sistemática da literatura.

\section{Tipos de estudos}

Foram incluídos estudos publicados em periódicos na forma completa, empregando delineamento de estudos transversais, coortes, casos-controle e ensaios clínicos randomizados (ECRs) publicados até 2016, com objetivo de identificar os principais estudos que avaliassem a relação entre o índice de massa corporal (IMC) e a função pulmonar em crianças e adolescentes. 


\section{Critérios de inclusão e exclusão}

Como critério de inclusão os estudos deveriam tratar-se de delineamentos em crianças e/ou adolescentes ( 0 a 20 anos), com ou sem diagnóstico de asma (hígidas ou asmáticas), sendo comparado ou associado à função pulmonar e ao índice de massa corporal (IMC). Como critério de exclusão considerou-se os estudos não disponíveis na íntegra, revisões (narrativa, sistemática ou metanálise) ou disponíveis em idiomas que não fossem inglês, espanhol ou português.

\section{Identificação dos estudos}

Um número de diferentes bases de dados eletrônicas foi utilizado para identificar os estudos publicados elegíveis, incluindo: PUBME, EMBASE, LILACS, SCIELO. A fim de facilitar a triagem dos estudos utilizou-se o software especifico para elaboração de revisões sistemáticas State of the Artthrough Systematic Review (StArt) (Hernandes et al., 2010). Mediante relação dos artigos pré-selecionados e importados para o StArt, dois pesquisadores, de forma independente, fizeram o rastreamento dos estudos duplicados, excluídos e potencialmente elegíveis à revisão, por meio da leitura dos títulos e resumos.

Em relação à relevância das escolhas dos estudos estabeleceram-se como critérios de seleção três etapas: (I) artigos selecionados igualmente pelos dois pesquisadores foram incluídos automaticamente; (II) artigos selecionados por apenas um pesquisador, foram analisados por terceiro pesquisador (revisor) e

(III) artigos não selecionados pelos dois pesquisadores ou por apenas um e revisado pelo terceiro pesquisador, sendo considerado não relevante, foram excluídos.

Estudos pré-selecionados (potencialmente elegíveis) foram avaliados na íntegra (leitura completa do artigo). Para finalização no processo de inclusão da sistemática foram avaliadas as referências dos estudos de revisões, bem como os elegíveis na tentativa de identificação de artigos não selecionados na busca das bases de dados correspondendo à pesquisa da literatura cinza.

\section{Termos da estratégia de pesquisa}

O levantamento nas bases de dados deu-se se utilizando a seguinte estratégia de busca: (( "Body Mass Index" OR BMI OR "Body Composition" OR "Body Fat Percentage" OR "Fat Percentage") AND Asthma AND ("Lung function" OR Spirometry)). Para fins de inclusão excessiva de artigos, todos unitermos foram selecionados e identificados nos três principais campos de pesquisa (title, abstract e keywords). Desta forma, os unitermos deveriam, obrigatoriamente, constar em pelo menos um dos três campos de pesquisa. Além deste, não foram aplicados filtros, tais como: data, idade, idioma, tipo de estudo, sendo identificados manualmente durante o processo de seleção pelos autores.

\section{Resultados}

No total, 744 artigos (729 por meio das bases de dados em saúde e 15 por meio das referências dos artigos elegíveis) foram pré-selecionados. Na primeira etapa foram excluídos 102 estudos duplicados e 573 por não se enquadrarem na temática da revisão sistemática. A partir dessa triagem, procedeu-se a leitura integral de 69 artigos, sendo excluídos 49 artigos por não contemplarem os critérios de inclusão (46) ou não estarem disponíveis para download (03), restando 20 artigos elegíveis para inclusão na revisão, conforme detalhado na Figura 1. 
Figura 1. Fluxograma da sistemática.

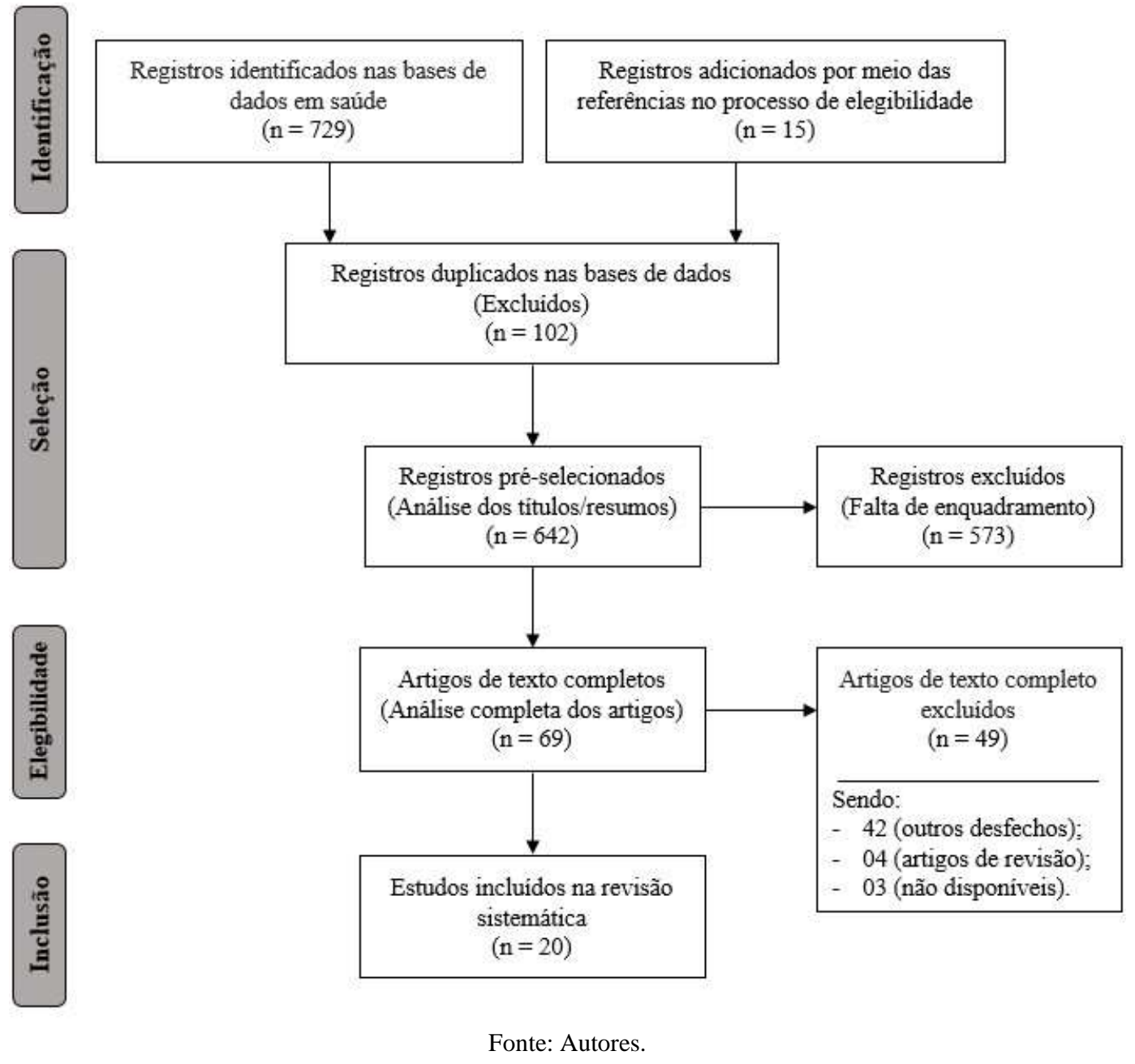

Dos 20 estudos selecionados, três foram conduzidos na América do Sul (Brasil), um na América Central (México), oito na América do Norte (Estados Unidos e Canadá), um na Oceania (Austrália), um no continente europeu (Grécia) e seis no continente asiático (China, Índia e Turquia). Quanto ao tipo de delineamento, 12 caracterizados como estudos transversais, cinco longitudinais (coortes), dois de caso-controle e um ensaio clínico randomizado, com idades mínimas de cinco e máximas de 20 anos, com uma amostra total de 41.924 crianças e adolescentes (Tabela 1). 
Tabela 1 - Caracterização dos estudos incluídos na revisão sistemática.

\begin{tabular}{|c|c|c|c|c|}
\hline Autores & Local & Delineamento & Faixa etária & Amostra \\
\hline Andrade et al., 2013 & Brasil & Transversal & 9 a 20 anos & 120 \\
\hline Assunção et al., 2014 & Brasil & Transversal & 8 a 18 anos & 59 \\
\hline Bekkers et al., 2013 & EstadosUnidos & Coorte & 8 a 12 anos & 1058 \\
\hline Bekkers et al., 2015 & EstadosUnidos & Coorte & 8 a 12 anos & 1090 \\
\hline Boran et al., 2007 & Turquia & Transversal & 7 a 15 anos & 130 \\
\hline Chen et al., 2009 & Canadá & Transversal & 6 a 17 anos & 718 \\
\hline Chow et al., 2009 & China & Transversal & 6 a 18 anos & 55 \\
\hline Chu et al., 2009 & China & Caso-controle & 13 a 16 anos & 14654 \\
\hline Eisenmann et al., 2007 & EstadosUnidos & Transversal & 6 a 12 anos & 256 \\
\hline Gold et al., 2003 & EstadosUnidos & Coorte & 6 a 14 anos & 9828 \\
\hline Han et al., 2014 & EstadosUnidos & Transversal & 6 a 17 anos & 2681 \\
\hline He et al., 2009 & China & Transversal & 8 a 12 anos & 2179 \\
\hline Jensen et al., 2014 & Austrália & Transversal & 8 a 17 anos & 48 \\
\hline Lang et al., 2011 & EstadosUnidos & Coorte & 6 a 16 anos & 420 \\
\hline Paralikar et al., 2012 & Índia & Caso-controle & 12 a 17 anos & 60 \\
\hline Pérez-Padilla et al., 2006 & México & Transversal & 8 a 20 anos & 6784 \\
\hline Rodrigues et al., 2007 & Brasil & Transversal & 6 a 18 anos & 20 \\
\hline Spathopoulos et al., 2009 & Grécia & Coorte & 6 a 11 anos & 657 \\
\hline Tantisira et al., 2003 & EstadosUnidos & ECR & 5 a 12 anos & 1039 \\
\hline Ulger et al., 2006 & Turquia & Transversal & 9 a 15 anos & 68 \\
\hline
\end{tabular}

Legenda: ECR: Ensaio clinico randomizado. Fonte: Elaborado pelos autores.

Doze estudos (60\%) tiveram como desfecho principal correlacionar o aumento IMC com a capacidade/volume pulmonar de crianças ou adolescentes com diagnóstico de asma. Do total, 10 (50\%) dos estudos não encontraram correlações entre o aumento do IMC e o volume expiratório forçado no primeiro minuto (VEF1). Dos que encontraram correlações, 5 (50\%) relataram haver correlação com a diminuição do VEF1 e os demais 5 (50\%) com o aumento do VEF1. Já para a capacidade vital forçada (CVF), do total, apenas 17 avaliaram a correlação entre o aumento do IMC, demonstrando que mais da metade $(9 ; 52,94 \%)$ não possui correlação com o desfecho (>IMC), e dos oito associados, seis tiveram correlação com o aumento da capacidade e apenas dois com a diminuição da CVF.

$\mathrm{Na}$ avaliação da "relação VEF1/CVF", dos 16 estudos que correlacionaram a variável (>IMC), sete (43,75\%) não encontraram associação com o VEF1/CVF e dos nove $(56,25 \%)$ que acharam correlação, oito (80\%) relataram haver diminuição de VEF1/CVF e apenas dois (20\%) haver aumento do VEF1/CVF. Por fim, apenas oito (40\%) estudos correlacionaram o >IMC com o FEF25-75\%, sendo relatado que na maioria (seis; 75\%) não houve correlação entre as duas variáveis e dois (25\%) apresentaram diminuição do FEF25-75\% ao aumento do IMC.

Dos 12 dos que avaliaram os desfechos entre grupos de asmáticos, sete (58,33\%) não apresentaram correlações entre o aumento do IMC e a diminuição da função pulmonar. Diferente dos demais (oito; 41,67\%) que avaliaram os mesmos desfechos, mas com grupos de hígidos, seis (75\%) apresentaram correlações entre o >IMC e a <FP, conforme demonstrado na Tabela 2 . 
Tabela 2 - Capacidades e volumes pulmonares, correlacionados ao aumento do Índice de Massa Corporal (IMC).

\begin{tabular}{|c|c|c|c|c|c|c|c|}
\hline Autores & Amostra & Grupos & VEF1 & CVF & VEF1/CV & FEF 25-75\% & IMC/FP \\
\hline Andrade et al., 2013 & 120 & $\dagger$ & $\# \varnothing$ & & & $\# \varnothing$ & - \\
\hline Assunção et al., 2014 & 59 & $\dagger$ & $\# \varnothing$ & $\# \varnothing$ & $\# \downarrow$ & & + \\
\hline Bekkers et al., 2013 & 1058 & $\dagger$ & $\# \varnothing$ & $\# \varnothing$ & & & - \\
\hline Bekkers et al., 2015 & 1090 & 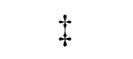 & $\# \varnothing$ & $\# \uparrow$ & $\# \downarrow$ & & + \\
\hline Boran et al., 2007 & 130 & $t$ & $\# \varnothing$ & $\# \varnothing$ & $\# \varnothing$ & & - \\
\hline Chen et al., 2009 & 718 & $t$ & $\# \varnothing$ & $\# \varnothing$ & $\# \varnothing$ & & - \\
\hline Chow et al., 2009 & 55 & $\dagger$ & $\# \downarrow$ & $\# \uparrow$ & $\# \varnothing$ & & + \\
\hline Chu et al., 2009 & 14654 & $\dagger$ & $\# \varnothing$ & $\# \varnothing$ & $\# \downarrow$ & $\# \varnothing$ & + \\
\hline Eisenmann et al., 2007 & 256 & $t$ & $\# \varnothing$ & $\# \varnothing$ & & $\# \varnothing$ & + \\
\hline Gold et al., 2003 & 9828 & $\dagger$ & $\# \varnothing$ & $\# \varnothing$ & $\# \varnothing$ & $\# \varnothing$ & - \\
\hline Han et al., 2014 & 2681 & $\dagger$ & $\# \uparrow$ & $\# \uparrow$ & $\# \downarrow$ & & - \\
\hline He et al., 2009 & 2179 & $\dagger$ & $\# \uparrow$ & $\# \uparrow$ & $\# \varnothing$ & & - \\
\hline Jensen et al., 2014 & 48 & $\dagger$ & $\# \uparrow$ & $\# \uparrow$ & $\# \varnothing$ & & - \\
\hline Lang et al., 2011 & 420 & $\dagger$ & $\# \downarrow$ & & $\# \downarrow$ & & + \\
\hline Paralikar et al., 2012 & 60 & $\ddagger$ & $\# \downarrow$ & $\# \varnothing$ & $\# \downarrow$ & $\# \varnothing$ & + \\
\hline Pérez-Padilla et al., 2006 & 6784 & $\dagger$ & $\# \downarrow$ & $\# \downarrow$ & $\# \uparrow$ & & + \\
\hline Rodrigues et al., 2007 & 20 & $\dagger$ & $\# \varnothing$ & & & $\# \varnothing$ & - \\
\hline Spathopoulos et al., 2009 & 657 & $t$ & $\# \downarrow$ & $\# \downarrow$ & $\# \downarrow$ & $\# \downarrow$ & + \\
\hline Tantisira et al., 2003 & 1039 & $\dagger$ & $\# \uparrow$ & $\# \uparrow$ & $\# \downarrow$ & & + \\
\hline Ulger et al., 2006 & 68 & $t$ & $\# \downarrow$ & $\# \downarrow$ & $\# \varnothing$ & $\# \downarrow$ & + \\
\hline
\end{tabular}

Legenda: CVF: Capacidade vital forçada; VEF1: Volume expiratório forçado no primeiro segundo; FEF25- 75\%: Fluxo expiratório forçado 25-75\%; VEF1/CVF: Volume expiratório forçado no primeiro segundo dividido pela capacidade vital forçada ("relação VEF1/CVF"); IMC/FP: Estudos que evidenciaram correlação entre o aumento do IMC e $\uparrow$ : Correlação entre grupos de asmáticos e hígidos; $\ddagger$ : Correlação entre grupos de hígidos eutróficos/sobrepeso/obesos; \#: Variáveis correlacionadas ao IMC nos estudos; $\emptyset$ : Variáveis sem correlação ao IMC; $\downarrow$ : Diminuição dos volumes/capacidades pulmonares; $\uparrow:$ Aumento dos volumes/capacidades pulmonares; +: Há correlação entre >IMC e diminuição da função pulmonar; -: Não há correlação entre >IMC e diminuição da função pulmonar; Fonte: Elaborado pelos autores.

\section{Discussão}

Marcadores antropométricos, como no caso do índice de massa corporal (IMC), são cada vez mais associados a fatores de riscos cardiovasculares e respiratórios. A correlação entre o aumento dos índices de adiposidade corpórea e doenças cardiovasculares são bem descritos na literatura, em virtude do aumento da prevalência da obesidade, trazendo consequências desde a infância até as fases finais da vida. Ao mesmo tempo, estudos associando o aumento dos índices de adiposidade corpórea e a diminuição dos volumes ou capacidades pulmonares até a atualidade não são bem estabelecidos, principalmente na população infantil, público este, que vem aumentando suas taxas de sobrepeso e obesidade anualmente.

Na presente revisão, buscou-se avaliar os principais estudos sobre o tema, tendo seus primeiros achados científicos publicados a partir de 2003 (Gold et al., 2003; Tantisira et al., 2003). O primeiro apresenta dados que associam a presença da asma à níveis elevados de IMC (Gold, 2003), enquanto o segundo relata um decréscimo nos valores de VEF1/CVF quando estes são correlacionados ao aumento do IMC (Tantisira, 2003).

A partir destes estudos, houve um crescente interesse sobre o tema por meio da comunidade científica. Dos 20 estudos elegíveis, apenas 11 (55\%) apresentaram correlações entre o aumento do IMC e a diminuição da função pulmonar em crianças e adolescentes (Quadro 2), demonstrando a pequena disposição de evidências sobre a correlação destes fatores. 
Assunção et al. (2014), conduziram um estudo para descrever os achados da função pulmonar em 59 crianças e adolescentes com excesso de peso sem doenças respiratórias. Os participantes foram submetidos à avaliação antropométrica, radiografia de tórax, oximetria de pulso, espirometria e medidas de volume pulmonar.

A análise descritiva dividiu a amostra em três grupos, dispostos da seguinte forma: sobrepeso: 4 componentes (7\%), obesos: 28 componentes (47\%) e severamente obesos: 27 componentes (46\%). Quanto à função pulmonar, apresentou-se dentro dos parâmetros fisiológicos em 21 participantes (36\%). Os demais, apresentam alterações ventilatórias, sendo que 32\% apresentaram doença obstrutiva, 25\% restritiva e $6 \%$ distúrbio ventilatório misto. A pesquisa evidenciou correlações inversas significativas entre os índices antropométricos e a relação VEF1/CVF nos indivíduos com distúrbio ventilatório misto. Os autores concluíram os participantes que tiveram a função pulmonar anormal estavam acima do peso, sugerindo que a obesidade afeta a função respiratória. Além disso, a proporção de resultados dentro dos valores de referência foi menor em indivíduos severamente obesos (Assunção, 2014).

Bekkers et al. (2015) conduziram uma coorte nos Estados Unidos, com 1090 crianças, a fim de analisar a associação entre IMC e circunferência da cintura (CC) com a função pulmonar de crianças asmáticas. A amostra foi subdivida em três categorias: abaixo do percentil 10, acima do percentil 90 e entre o percentil 10 e 90 . No estudo foi considerado a CVF como medida de volume pulmonar, o FEV1 como a permeabilidade das vias aéreas e a relação como indicadora de obstrução em vias aéreas. Em meninos, o resultado sugere associações inversas entre IMC e CC com CVF e VEF1. Nas análises de regressão, as crianças com IMC elevado tiveram maior CVF do que as crianças com IMC normal, sendo que as meninas com IMC elevado apresentaram valores 4,6\% maiores para CVF. A relação VEF1/CVF foi significativamente menor nos indivíduos com sobrepeso. O estudo concluiu que crianças com IMC elevado possuem maior volume pulmonar (CVF) do que as crianças com IMC normal.

Chow et al. (2009), investigou a associação entre obesidade e inflamação das vias aéreas e dos parâmetros espirométricos em 55 crianças e adolescentes com e sem diagnóstico de asma. $\mathrm{Na}$ análise dos resultados constatou-se que o VEF1 e CVF foram significativamente diferentes entre os sujeitos $(\mathrm{p}=0,040$ e $\mathrm{p}=0,005)$. O peso para a altura $\mathrm{z}$-score correlacionou-se positivamente com a CVF $(\mathrm{p}=0,041)$, mas inversamente com o VEF1/CVF ( $\mathrm{p}=0,049)$, sendo queessas correlações não foram observadas em crianças asmáticas. Os autores concluíram o estudo relatando haver diferença significativa apenas para os parâmetros de VEF1 em crianças e adolescentes.

Chu et al. (2009) realizaram um estudo caso-controle na China, com 14.654 crianças e adolescentes, com objetivo de determinar se a prevalência de asma difere entre crianças obesas e não obesas. Em relação à avaliação antropométrica considerou-se sobrepeso aqueles com percentil de IMC entre 85-95\% e obeso quando o percentil de IMC superior a 95\%. Foi observado que a prevalência de asma aumentou com o IMC elevado e que o VEF1/CVF diminuiu à medida que o IMC aumentou, em ambos os sexos no grupo de obesos e com sobrepeso no grupo não-asmático. O estudo mostrou que o IMC elevado em ambos os sexos foi associado com baixo VEF1/CVF e um IMC baixo nos meninos pode estar associado com uma baixa $\mathrm{FEF} 25-75 \%$, além de contribuir para os sintomas da asma.

Eisenmann et al. (2007) buscou avaliar a influência da obesidade na função pulmonar em crianças de 6 a 12 anos em duas reservas indígenas da América do Norte. A amostra foi constituída por 813 crianças. Após análise estatística constatou-se que cerca de $26 \%$ das crianças foram definidas como sobrepeso e $16 \%$ como obesos. Os achados do estudo indicam um aumento da função pulmonar entre eutróficos e com sobrepeso e uma diminuição em crianças obesas, sendo sugerido haver consequências na função pulmonar de crianças obesas.

Lang et al. (2011) conduziram uma coorte de pacientes asmáticos a fim de determinar os impactos da idade e a relação entre obesidade e parâmetros espirométricos. A amostra consistiu em 490 indivíduos asmáticos. Após análise das variáveis 
observou-se que o efeito da obesidade e da asma muda com a idade e com o sexo, sendo que, os obesos tiveram maior redução da função pulmonar, porém, com menos sintomas de asma do que os asmáticos não- obesos semelhantes. Obesos entre 12 e 17 anos tiveram uma tendência para maiores sintomas de obstrução do fluxo de ar e asma, em comparação com os asmáticos nãoobesos. Assim, sugere-se que a idade possui efeito modelador significativo sobre a relação entre a obesidade e o fenótipo da asma. Com o aumento da idade, a influência da obesidade sobre o fenótipo da asma é geralmente reduzida. Na faixa etária de 6 a 11 anos de idade percebeu-se diminuição do VEF1 e do VEF1/CVF à medida que o IMC aumentou, indicando que crianças pareciam estar mais afetadas pela obesidade em termos de função pulmonar e foi o único grupo com evidências de obstrução ao fluxo aéreo relacionada com a obesidade. Os autores afirmam ainda que o fenótipo da asma possa ser mais afetado pela obesidade entre crianças e mulheres.

Paralikar et al. (2015) realizaram um estudo caso-controle com 60 crianças e adolescentes do sexo masculino com objetivo de avaliar a função pulmonar de obesos e comparar com parâmetros antropométricos. Meninos com IMC acima do percentil 95 para a idade foram considerados obesos e os demais controles. Tanto VEF1 quanto VEF1/CVF foram significativamente reduzidos no grupo de obesos $(p<0,01)$. No entanto, nenhuma dificuldade obstrutiva foi detectada em qualquer sujeito do grupo obeso. Quanto a CVF e as taxas de fluxo (PFE e FEF25-75\%), não houve diferença significativa entre os grupos. Desta forma, conclui-se que as funções pulmonares estão diminuídas (em comparação com indivíduos com peso normal) no grupo de meninos adolescentes obesos.

Pérez-Padilla et al. (2006) conduziram um estudo transversal com 6.784 crianças e adolescentes entre 8 e 20 anos de idade, a fim de descrever a prevalência de obesidade entre as crianças mexicanas e mexicanas-americanas sem asma e seu impacto na função pulmonar. Estimou-se a prevalência da obesidade percentil >95. A partir da análise dos dados dos 6784 participantes, 4,2\% relataram diagnóstico médico de asma no questionário, quanto a função pulmonar o VEF1 e a CVF foram ligeiramente mais baixos nos mexicano-americanos (124 ml e $129 \mathrm{ml}$, em média, respectivamente). Entretanto, tais achados modificaram-se com a idade visto que, a associação entre a função ventilatória e IMC foi diferente aos 12 anos. Nos primeiros anos estudados, CVF e VEF1 aumentaram em indivíduos com maior índice de massa corporal. Mas em sujeitos >12 anos de idade, o padrão foi invertido, especialmente para VEF1 em meninos tanto mexicanos como mexicano- americanos, sendo concluído que a função pulmonar é negativamente influenciada pelo sobrepeso e obesidade, principalmente nos jovens.

Spathopoulos et al. (2009) realizaram uma coorte com indivíduos de 6 a 11 anos de idade para investigar o efeito da obesidade sobre a função pulmonar em crianças eutróficas, com sobrepeso e obesas. A amostra foi composta por 853 participantes que realizaram avaliação antropométrica e testes de função pulmonar sendo subdivididos em 357 com sobrepeso, 300 obesos e 196 eutróficos. Após análise estatística percebeu-se que os parâmetros espirométricos (CVF, VEF1, FEF25-75\% e VEF1/CVF) foram significativamente reduzidos em crianças com sobrepeso ou obesos em comparação com crianças com peso normal ( $\mathrm{p}=0,007, \mathrm{p}<0,001, \mathrm{p}<0,001 \mathrm{e} \mathrm{p}<0,001$, respectivamente). Os autores evidenciaram que o IMC elevado é um forte fator de risco independente para a asma $(\mathrm{OR}=2,17,95 \% \mathrm{CI}=1,22-3,87, p=0,009)$ e para atopia $(\mathrm{OR}=2,06,95 \% \mathrm{CI}=$ 1,32-3,22, $p=0,002)$. O efeito do aumento do IMC sobre a asma foi significativo em crianças do sexo feminino, mas não para o sexo masculino $(\mathrm{OR}=2,73,95 \% \mathrm{CI}=1,09-6,85, p=0,032$; $\mathrm{OR}=1,74,95 \% \mathrm{CI}=0,83-3,73, p=0,137$, respectivamente). Em conclusão, os dados sugerem que o IMC elevado pode ser um determinante importante de parâmetros de espirometria reduzidos bem como um fator de risco para a asma em crianças do sexo feminino.

Tantisira et al. (2003) conduziram uma pesquisa a fim de testar a hipótese de que o IMC seria independentemente associado com medidas de gravidade da asma em uma população de crianças com asma leve a moderada. A amostra do estudo foi oriunda do estudo Childhood Asthma Management Program (CAMP) de delineamento transversal que incluiu 1041 crianças com idade entre 5 e 12 anos. Em relação aos resultados sobre a relação entre IMC e função pulmonar, o aumento do 
IMC foi associado com o aumento da VEF1 $(\mathrm{p}=0,006)$ e da CVF $(\mathrm{p}=0,012)$. No entanto, decréscimos na relação VEF1/CVF

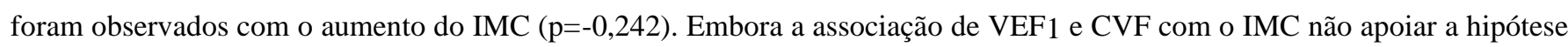
inicial do estudo, os autores concluíram que o decréscimo observado na relação VEF1/CVF tem potencial relevância na relação entre IMC e a gravidade da asma.

Ulger et al. (2006) realizaram um estudo a fim de investigar o efeito da obesidade na função pulmonar, bem como para detectar a incidência de hiperresponsividade das vias aéreas e broncoespasmo induzido pelo exercício em 38 crianças e adolescentes. Após análise estatística constatou-se que os testes de função respiratória basal foram menores no grupo de estudo e o teste de exercício foi positivo em 31,6\% do grupo de obesos e em 3,3\% do grupo controle ( $\mathrm{p}=0,003)$. Além disso, observou-se fortes correlações negativas entre o índice de massa corporal (IMC), peso relativo, espessura da prega cutânea, relação cintura/circunferência do quadril e CVF, VEF1, $\mathrm{FEF}_{25-75 \%}$ e pico de fluxo expiratório (PEF). Os autores sugerem com o estudo que o IMC possui correlação negativa para as variáveis CVF, VEF1, FEF25-75\% e pico de fluxo expiratório (PEF).

Contudo, o restante dos estudos avaliados concluiu que os parâmetros antropométricos não mostraram efeito significativo na função pulmonar, sendo necessários mais estudos longitudinais para explorar os efeitos da obesidade na função pulmonar das crianças, visto que, tais variáveis podem mudar com o passar do tempo, podendo sofrer influências ambientais (Gold, 2003; Boran, 2007; Rodrigues, 2007; He, 2009; Andrade, 2013; Bekkers, 2013; Jensen, 2013; Han et al., 2014). Outros autores relatam haver maior relação da diminuição dos valores pulmonares com outras medidas antropométricas, tais como a circunferência da cintura (CC), visto que esta região corporal é a de maior prevalência no acúmulo adiposo em seres humanos (Chen, 2009; Bekkers, 2013; Han et al., 2014).

Dos 20 estudos analisados, 12 estudaram a correlação de desfecho entre grupos de asmáticos vs. hígidos. Destes, 7 não apresentaram correlações entre as variáveis de desfecho, diferentemente dos demais, que avaliaram os mesmos desfechos entre grupos de hígidos (eutrófico, sobrepeso, obeso), onde, 75\% apresentou correlação inversa entre o IMC e a FP. Isso demonstra que deve haver maior associação entre grupos de hígidos do que entre os grupos de asmáticos vs. hígidos, indicando que não há associação entre asma e diminuição da função pulmonar pelo acúmulo de adiposidade corpórea em crianças e adolescentes.

A principal limitação da presente revisão foi a falta de padronização e a homogeneidade dos valores da função pulmonar nas crianças e adolescentes. Desta forma, não foi possível realizar uma metanálise dos achados para uma melhor compreensão e busca de evidências científicas quantitativas sobre a correlação entre as variáveis IMC e função pulmonar na população infantil.

\section{Conclusão}

Mesmo sendo apontados por grande parte dos estudos que o aumento do IMC tende a uma diminuição de volumes e capacidades pulmonares, estes devem ser avaliados com cautela, pois uma parcela considerável dos trabalhos analisados não demonstrou correlação entre os desfechos avaliados, contestando esta evidência. A falta de consenso entre os achados pode estar associada à diversidade de métodos e delineamentos aplicados, devendo haver uma padronização entre a causa e o efeito.

\section{Referências}

Abeso (2009). Associação Brasileira para Estudo da Obesidade e Síndrome Metabólica de Obesidade DB. AC Farmacêutica.

Alwan A. (2011). Global status report on non communicable diseases 2010: World Health Organization;

Andrade, L. S., Araújo, A. C. T., Cauduro, T. M., Watanabe, L. A., Castro, A. P., \& Jacob, C. M. A., et al. (2013). Obesidade e asma: associação ou epifenômeno? Revista Paulista de Pediatria.; 31(2): 138-44.

Assunção, S. N. F., Daltro, C. H. D. C., Sorte, B., Christian, N., Júnior, R., \& da Costa, H., et al. (2014). Lung function in the absence of respiratory symptoms in overweight children and adolescents. Jornal Brasileiro de Pneumologia. 40(2): 134-41. 
Bekkers, M., Wijga, A. H., de Jongste, J. C., Kerkhof, M., Postma, D., \& Gehring, U., et al. (2013). Waist circumference, BMI, and lung function in 8-year-old children: The PIAMA birth cohort study. Pediatric pulmonology.48 (7):674-82.

Bekkers, M. B., Wijga, A. H., Gehring, U., Koppelman, G. H., de Jongste, J. C., \& Smit, H. A., et al. (2015). BMI, waist circumference at 8 and 12 years of age and FVC and FEV(1) at 12 years of age; the PIAMA birth cohort study. BMC Pulm Med.15. London.

Boran, P., Tokuc, G., Pisgin, B., Oktem, S., Yegin, Z., \& Bostan, O. (2007). Impact of obesity on ventilatory function. Jornal de pediatria. 83(2):171-6.

Chen, Y., Rennie, D., Cormier, Y., \& Dosman, J. A. (2009). Waist circumference associated with pulmonary function in children. Pediatric pulmonology.; 44(3): 216-21.

Chow, J., Leung, A., Li, W., Tse, T., Sy, H., \& Leung, T. (2009). Airway inflammatory and spirometric measurements in obese children. Hong Kong medical journal = Xianggangyixuezazhi / Hong Kong Academy of Medicine.15(5): 346-52.

Chu, Y. T., Chen, W. Y., Wang, T. N., Tseng, H. I., Wu, J. R., \& Ko, Y. C. (2009). Extreme BMI predicts higher asthma prevalence and is associated with lung function impairment in school-aged children. Pediatric pulmonology.; 44(5): 472-9.

Eisenmann, J. C., Arnall, D. A., Kanuho, V., Interpretter, C., \& Coast, J. R. (2007). Obesity and pulmonary function in Navajo and Hopi children. Ethnicity \& disease.17(1): 14-8.

Gold, D. R., Damokosh, A. I., Dockery, D. W., \& Berkey, C. S. (2003). Body-mass index as a predictor of incident asthma in a prospective cohort of children. Pediatric pulmonology. 36 (6): 514-21.

Han, Y-Y, Forno, E., \& Celedón, J. C. (2014). Adiposity, fractional exhaled nitric oxide, and asthma in US children. American journal of respiratory and critical care medicine. 190(1): 32-9.

He, Q. Q., Wong, T. W., Du, L., Jiang, Z. Q., Qiu, H., \& Gao, Y., et al. (2009). Respiratory health in overweight and obese Chinese children. Pediatric pulmonology. 44(10): 997-1002.

Hernandes, E., Zamboni, A., \& Di Thommazo, A (2010) editors. StArt Uma Ferramenta Computacional de Apoio à Revisão Sistemática. Congresso Brasileiro de Software (CBSoft'10), Salvador, Brazil.

Jensen, M. E., Gibson, P. G., Collins, C. E., \& Wood, L. G. (2013). Lean mass, not fat mass, is associated with lung function in male and female children with asthma. Pediatric research.75(1-1):93-8.

Koenig, S. M. (2001). Pulmonary complications of obesity. The American journal of the medical sciences; 321(4): 249-79.

Lang, J. E., Hossain, J., Dixon, A. E., Shade, D., Wise, R. A., \& Peters, S. P., et al. (2012). Does Age Impact the Obese Asthma Phenotype? CHEST 2011; 140 (6): 1524- 33 .

Paralikar, S. J., Kathrotia, R. G., Pathak, N. R., \& Jani, M. B. (2012). Assessment of pulmonary functions in obese adolescent boys. Lung India: Official Organ of Indian Chest Society. 29(3):236-40.

Pérez-Padilla, R., Rojas, R., Torres, V., Borja-Aburto, V., Olaiz, G., \& Empece Working, G. (2006). Obesity among children residing in Mexico City and its impact on lung function: a comparison with Mexican-Americans. Archives of medical research. 37(1): 165-71.

Rodrigues, J. C., Takahashi, A., Olmos, F. M. A., Souza, J. B., Bussamra, M. H. F., \& Cardieri, J. M. A. (2007). Efeito do índice de massa corpórea na gravidade da asma e na reatividade brônquica induzida pelo exercício em criançasasmáticas com sobrepeso e obesas. Rev Paul Pediatr.;25(3):207-13.

Spathopoulos D, Paraskakis E, Trypsianis G, Tsalkidis A, Arvanitidou V \& Emporiadou M, et al. (2009). The effect of obesity on pulmonary lung function of school aged children in Greece. Pediatric pulmonology. 44(3): 273- 80.

Schwimmer J. B., Burwinkle T. M. \& Varni J. W. (2003). Health-related quality of life of severely obese children and adolescents. Jama. 289(14):1813-9.

Sigulem D. M., Taddei J, Escrivão M., \& Devincenzi M. U. (2001). Obesidade na infância e na adolescência. Compacta Nutr 2:5-16.

Suchindran C., North K. E., Popkin B. M., \& Gordon-Larsen P. (2010). Association of adolescent obesity with risk of severe obesity in adulthood. Jama.; 304(18):2042-7.

Tantisira K, Litonjua A, Weiss S., \& Fuhlbrigge A. (2003). Association of body mass with pulmonary function in the Childhood Asthma Management Program (CAMP). Thorax.58(12):1036-41.

Ulger Z, Demir E, Tanac R, Goksen D, Gulen F \& Darcan S, et al. (2006). The effect of childhood obesity on respiratory function tests and airway hyperresponsiveness. The Turkish journal of pediatrics; 48 (1): 43-50. 